\title{
Tough Polylactide Blend System for Osteosynthesis Procedures
}

\author{
Jone Muñoz ${ }^{1,2}$, Eider Aldalur', Eva Sanchez-Rexach ${ }^{1,2}$, Iñigo Revilla1, \\ Aitor Larrañaga ${ }^{1,2}$, Jose-Ramon Sarasua ${ }^{1,2}$ \\ ${ }^{1}$ Department of Mining-Metallurgy Engineering and Materials Science, School of Engineering \\ University of the Basque Country (UPV-EHU) \\ Plaza Ingeniero Torres Quevedo 1, 48013, Bilbao \\ ${ }^{2}$ Basque Excellence Research Center in Macromolecular Design \& Engineering, POLYMAT \\ $\mathrm{P}^{\circ}$ Manuel de Lardizabal 3, 20009 Donostia-San Sebastián, Spain \\ jone.munoz@ehu.eus; jr.sarasua@ehu.eus
}

\begin{abstract}
This work stems from the need to contribute, improve and collaborate in the design of new bioresorbable polymeric implants for bone fracture fixations (osteosynthesis procedures), particularly those involving polylactides (PLA). Choosing materials for orthopedic implants often imposes strict requirements for the mechanical properties. PLA is widely employed for this purpose; however toughening schemes are necessary for its suitable application. Blending of poly(D,L-lactide) (PDLLA) and biodegradable poly(L-lactideco- $\varepsilon$-caprolactone) copolymer (PLCL) showing a thermoplastic-elastomeric behavior has been performed in an effort to toughen the PDLLA without compromising its biodegradability and biocompatibility. The miscibility state of PDLLA/PLCL blend system is studied by means of Differential Scanning Calorimetry (DSC), phase morphology is observed by Scanning Electron Microscopy (SEM) and eventually, the changes in mechanical properties during the first 3 months at body temperature is also discussed to establish a preliminary outline of the response that could be expected from this system in service. Blends of PDLLA/PLCL show two clear $T_{g}$ indicating immiscibility of the blends. The phase separation is also corroborated by SEM micrographs where two distinct phases are observed clearly, one acting as a matrix and the other one as a dispersed phase. Initially, the toughness of PDLLA was considerably improved by the addition of PLCL. The elongation at break was significantly increased. However, the mechanical properties loss during the aging at body temperature of the blends indicates the unsuitability of these blends to use as bone fracture fixations.
\end{abstract}

Keywords: Fracture fixation implants, Poly(D,L-Lactide), Poly(L-lactide-co-e-caprolactone), Polymer Blends, Toughening, Aging, Mechanical Properties.

\section{Introduction}

Bioabsorbable internal fixation of hard and soft tissue has gained popularity over the past 20 years, especially in the fields of sports medicine, trauma and craniofacial surgery. State-of-the-art materials in the mid-20th Century for osteosythesis included metals such as stainless steel and others. While successful, bone near the metal implant can become osteoporotic due to stress-shielding effect [1] leading occasionally to bone fracture after the plate removal [2]. Other complications include metal sensitivity, tissue irritation, mutagenicity [3] and the fact that metal implants have to be removed after complete bone healing. Therefore, another surgical intervention is necessary with additional risk and cost. To avoid the stress-shielding, it is desirable to use implants made of materials with mechanical properties close to those of the bone. The best candidates are polymers, especially the biodegradable ones that would not cause long term foreign particle accumulation in the body and would eliminate the cost and discomfort of a removal surgery. Therefore, studies on such biodegradable polymeric osteofixation devices have been continuing since the introduction of first biodegradable devices in 1966 [4].

Usually high mechanical strength and stiffness are extremely important in designing biodegradable devices, such as, screws, plates or pins, for regenerative hard tissue procedures. In this sense, polylactides (PLAs) and their composites are one of the most frequently adopted biodegradable polymers in bone fixation systems [5]-[10]. PLA is a high strength and high modulus thermoplastic, which can be easily processed by conventional processing techniques like injection moulding, blow moulding, thermoforming and extrusion [11]. One of the prominent features of PLAs chemistry is derived from the chirality of the monomer molecules. Lactide presents two asymmetrical carbon atoms giving rise to two optically active configurations; poly(L-lactide) (PLLA) and poly(D-Lactide) (PDLA). These stereo-regular polymers are semicrystalline 
with a glass transition temperature $\left(\mathrm{T}_{\mathrm{g}}\right) \sim 60{ }^{\circ} \mathrm{C}$ and melting temperature $\left(\mathrm{T}_{\mathrm{m}}\right) \sim 180{ }^{\circ} \mathrm{C}$. However, when both enantiomers of opposite configuration (LL/DD) are irregularly distributed within the polylactide backbone, the chain microstructure turns out to be stereo-irregular and then polylactides can be regarded as optically non-pure copolymers termed poly(D,L-lactide) (PDLLA). When the same amount of LL and DD units are equimolar and randomly incorporated into the polylactide chain microstructure a completely amorphous PDLLA with a $\mathrm{T}_{\mathrm{g}}$ around $55{ }^{\circ} \mathrm{C}$ is obtained [12]. This work focused on PDLLA. Despite the fact that PDLLA shows suitable elastic modulus and mechanical strength, its inherent brittleness when processed using standard processing conditions [13], limits its use in the above mentioned applications.

From a clinical point of view, a ductile mode of deformation is desirable, since a brittle breakage of an implant before union of a fracture results in bone redisplacement and loose of material fragments, which might cause irritation or some adverse reaction [14]-[16]. Therefore, in order for PLAs to be suitable for applications where mechanical toughness is required, toughening schemes and improved composites or blends are needed. This strategy will improve the functionality and reliability of PLA based implants, and in turn leading to more stable surgical processes.

Many strategies have been developed to alter the properties of a polymer. The chemical synthesis of copolymers is an interesting method in order to design new polymers with special properties. However, this method is generally more expensive, as well as, less-industrial-practiced method than the blending operation. A proper selection and combination of polymeric components in a certain ratio might result in a blend material with optimal properties for a specific application. In this work, PDLLA was blended with a biodegradable and bioresorbable poly(L-lactide-co- $\square \square$-caprolactone) copolymer (PLCL) to improve the toughness. The PLCL selected has a molar composition of approximately $70 \%$ L-lactide and $30 \%$ of $\varepsilon$-caprolactone. It is an amorphous polymer with a $\mathrm{T}_{\mathrm{g}}$ of $\sim 24^{\circ} \mathrm{C}$ and presents a thermoplastic-elastomeric character, i.e., elastomeric behavior, high elongation at break and high elastic recovery capacity. Moreover, in general, PLCLs offer better thermal stability during processing as well as a suitable toughness for being used as raw material in the design of implants and medical devices [[17]-[19]]. These characteristics can provide an improvement in the mechanical properties of PDLLA.

In this work, a polymer blend system composed of PDLLA and PLCL copolymer, PDLLA/PLCL is presented as an alternative biodegradable and bioresorbable material to tune the properties of PDLLA. The aim of this work is to study the miscibility state, phase behavior and mechanical properties of this polymeric system. Eventually, the changes in mechanical properties during the first 3 months at body temperature is also studied to establish a preliminary outline of the response that could be expected from this system in service.

\section{Materials and Methods \\ 2.1. Materials}

Poly(D,L-lactide) (PDLLA) was supplied by Purac (The Netherlands). The number average molecular weight $\left(\mathrm{M}_{\mathrm{n}}\right)$ and polydispersity index (D) were $28000 \mathrm{~g} \mathrm{~mol}^{-1}$ and 2.0 , respectively.

Poly(L-lactide-co- $\varepsilon$-caprolactone) (PLCL) of approx. 70:30 L-lactide/ $\varepsilon$-caprolactone in molar ratio was supplied by Purac Biochem (The Netherlands). The number average molecular weight $\left(\mathrm{M}_{\mathrm{n}}\right)$ and polydispersity index (D) measured were $105000 \mathrm{~g} \mathrm{~mol}^{-1}$ and 1.68 , respectively.

In both cases, the molecular weight distribution was analyzed by gel permeation chromatography using a Waters 1515 chromatograph apparatus equipped with a Waters 2414 refractive index (RI) detector. Chloroform at $35^{\circ} \mathrm{C}$ was used as the eluent and the Styragel columns were calibrated with polystryrene standards.

\subsection{Blend Preparation and Aging Process}

Sheets of neat polymers and their blends were prepared by melt-mixing by Brabender Plasti-Corder OHG (Germany) at $200{ }^{\circ} \mathrm{C}$ and then conformed by compression moulding in a Collin P $200 \mathrm{E}$ hydraulic press (Germany) at $200{ }^{\circ} \mathrm{C}$ followed by water quenching. The resulting sheets had a thickness of $1 \mathrm{~mm}$.

Then, the moulded sheets were treated at constant temperature of $37^{\circ} \mathrm{C}$ and relative humidity (RH) of $40 \%$ in a climatic chamber (Dycometal) for different periods of time (1, 2, 3, months).

\subsection{Determination of Polymer Blend Miscibility}

The miscibility state of the blend system was evaluated according to the unique glass transition temperature $\left(T_{\mathrm{g}}\right)$ criterion. The most common use of $\mathrm{T}_{\mathrm{g}}$ in determination of polymer/polymer miscibility is based on the premise that a single 
$\mathrm{T}_{\mathrm{g}}$ indicates the domain size is comparable to the macromolecular radius of gyration, i.e., $2 \leq \mathrm{d}_{\mathrm{d}} \leq 15 \mathrm{~nm}$, which is necessary requisite for fulfilling the condition of the thermodynamic micibility. Therefore, following this criterion it is accepted, that blends displaying a single $\mathrm{T}_{\mathrm{g}}$ are miscible [20]. The $\mathrm{T}_{\mathrm{g}}$ behavior was analyzed by means of Differential Scanning Calorimetry (DSC).

Thermal behavior was analyzed by a Q200-Differential Scanning Calorimeter (TA Instruments), calibrated with pure indium and sapphires. Two subsequent scans were performed from $-60{ }^{\circ} \mathrm{C}$ to $200{ }^{\circ} \mathrm{C}$ at $20^{\circ} \mathrm{C} \mathrm{min}{ }^{-1}$. Thermal properties were measured in the second scan. The glass transition temperature $\left(\mathrm{T}_{\mathrm{g}}\right)$ was determined from the inflection point of the heat flow curve, the cold crystallization temperature $\left(\mathrm{T}_{\mathrm{c}}\right)$ and the melting temperature $\left(\mathrm{T}_{\mathrm{m}}\right)$ from the exothermic and endothermic peak position, respectively, and the cold crystallization enthalpy $\left(\Delta \mathrm{H}_{\mathrm{c}}\right)$ and melt enthalpy $\left(\Delta \mathrm{H}_{\mathrm{m}}\right)$ were obtained calculating the area under the corresponding crystallization and melting peaks. The sample weight was between 5 and $10 \mathrm{mg}$ in all cases.

\subsection{Phase Morphology}

A JSM-6400 (JEOL) Scanning Electronic Microscope was used to observe the fracture surfaces of the broken specimens after tensile tests and analyze phase morphology of the blends. Previous to observation the polymeric specimens were coated with a thin layer of gold using a BAL-TEC SCD 004 sputter coater.

\subsection{Mechanical Properties}

Tensile tests were performed at $22{ }^{\circ} \mathrm{C}$ and $50 \%$ of relative humidity $(\mathrm{RH})$ with an Instron 5565 testing machine at a crosshead speed of $5 \mathrm{~mm} \mathrm{~min}^{-1}$. Dumbell-shaped samples were punched out from sheets following ISO 527-2 (ISO 527-2 /5A/ 5).

\section{Results and Discussion}

The miscibility of any blend system mainly depends on the behavior of the blend components. Three different miscibility behaviors can be expected in any polymeric blend system: The components demix at all blend compositions in the pure components (fully immiscible); the components exhibit phase separation with partial solubility of the components or a miscibility gap depending on composition (partially miscible), or the components form an homogeneous phase in the whole compositional range (fully miscible).

Blends of poly(D,L-Lactide) (PDLLA) and Poly(L-lactide-co- $\varepsilon$-caprolactone) (PLCL) show two well defined glass transition temperatures $\left(\mathrm{T}_{\mathrm{g}}\right)$ in the DSC traces as can be observed in Fig. 1. The fist $\mathrm{T}_{\mathrm{g} 1}$ around $25^{\circ} \mathrm{C}$ corresponds to pure PLCL copolymer while the second $\mathrm{T}_{\mathrm{g} 2}$ around $54{ }^{\circ} \mathrm{C}$ corresponds to pure PDLLA. This result indicate that PDLLA/PLCL blend system is fully immiscible. It is known that immiscible binary blend generally leads to insignificant improvements in mechanical properties, like shown by polymer blends of polylactides containing polycaprolactone (PCL) [[13], [21]-[25]]. However, in the present PDLLA/PLCL system the initial mechanical properties improve in terms of toughening and ductility without any decrease in the mechanical resistance. 


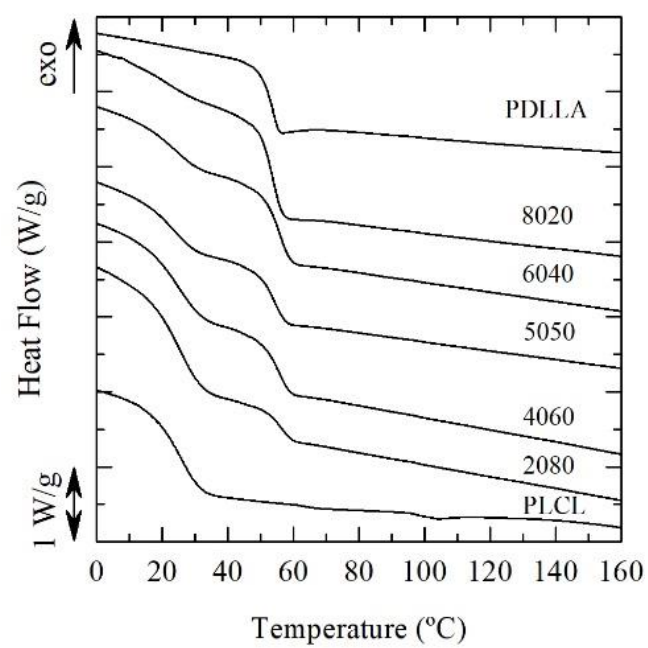

(a)

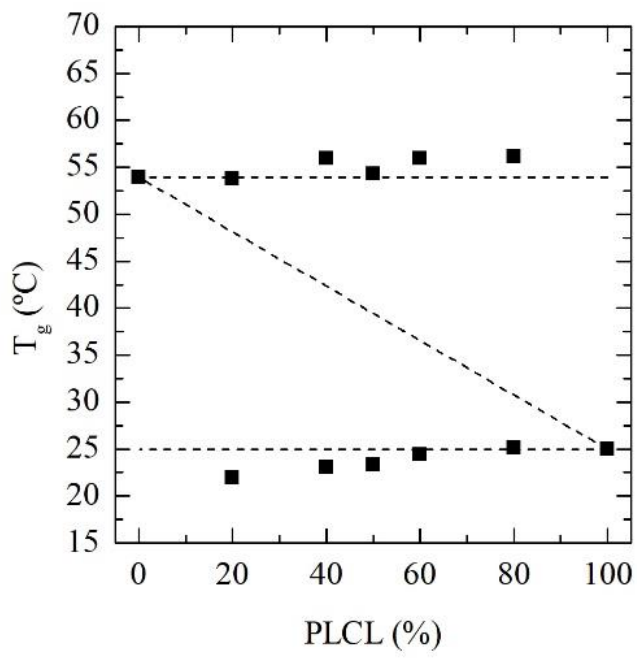

(b)

Fig. 1: (a) $2^{\text {nd }}$ scan DSC traces for PDLLA, PLCL and PDLLA/PLCL blends of different compositions (b) $T_{g}$ behavior depending on PLCL copolymer content for PDLLA/PLCL blend system. The horizontal lines represent the behavior of a fully immiscible polymer blend.

The stiffness, the strength and the elongation at break of the melt-quenched PDLLA/PLCL blends were analyzed by means of tensile tests. Fig. 2.a shows the representative tensile curves of pure PDLLA, PLCL and its blends at different compositions. Pure PDLLA displays a brittle fracture showing a failure immediately upon the tensile load. Its tensile strength is $34 \mathrm{MPa}$, while elongation at break is only $4 \%$. On the contrary, PLCL behaves as an elastomeric material exhibiting a large elongation at break of $837 \%$. As it is shown in Fig. 2, the addition of PLCL changed clearly the mechanical behavior and improved considerably the toughness of the PDLLA increasing significantly the elongation before it breaks. For example, $\sigma_{\max }$ of compositions of $20 \mathrm{wt} . \%$ and $40 \mathrm{wt} . \%$ of PLCL is $38 \mathrm{MPa}$ and $36 \mathrm{MPa}$, respectively, but the elongation at break increases significantly showing values much higher than that of pure PDLLA, $314 \%$ and $452 \%$, respectively (see Table 1 ). In general, the elongation at break increases with increasing PLCL content as shown in Fig. 2.b, while the tensile strength remains constant and the elastic modulus decrease with the addition of the copolymer.

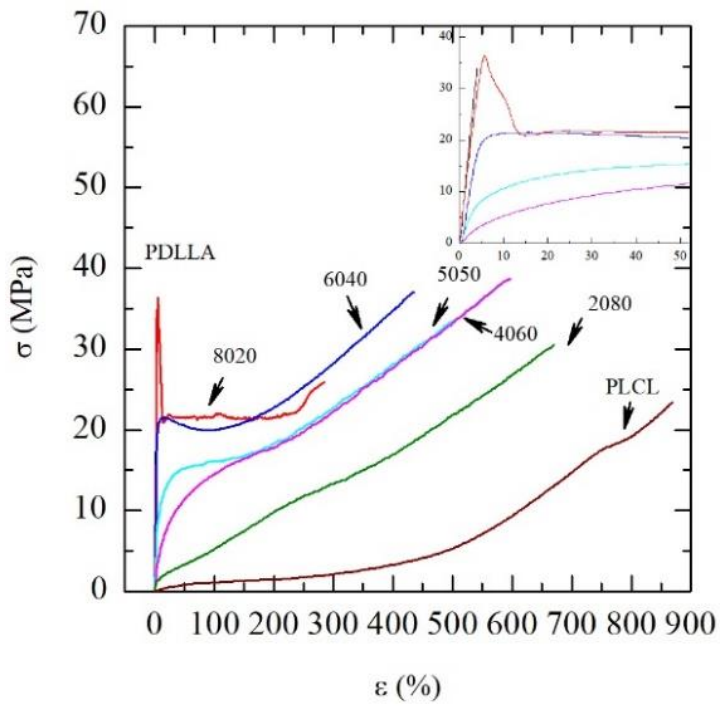

(a)

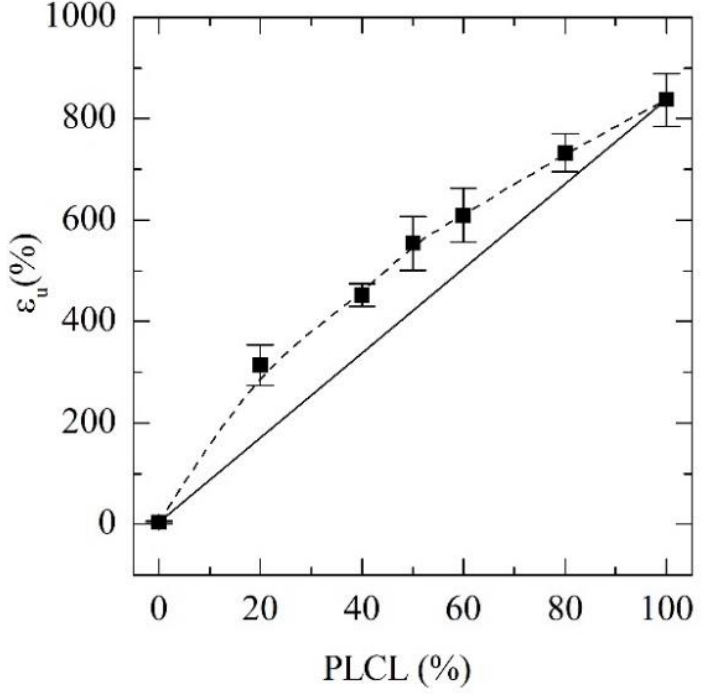

(b)

Fig. 2: For unaged PDLLA/PLCL blend system (a) Representative stress-strain curves and (b) Ultimate elongation at break. 
The preliminary results obtained with respect to mechanical properties of PDLLA/PLCL blend system were quite positive for designing and improving orthopaedic implants for bone fracture fixations, however the changes in those parameters have to be studied at service conditions in order to assess whether this polymeric system is suitable or not for these applications. For this purpose, PDLLA/PLCL melt-quenched sheets were subjected to aging or an annealing process at body temperature, $37^{\circ} \mathrm{C}$, for 1,2 , and 3 months for each blend composition. Table 1 summarizes the most relevant mechanical properties including the elastic modulus (E), the tensile strength $\left(\sigma_{\max }\right)$, strain or elongation at break point $\left(\square_{\mathrm{u}}\right)$ for unaged and aged blend samples (the mechanical properties of samples aged for 3 months could not be measured due to their brittleness).

Table 1: Mechanical properties of neat PDLLA, PLCL and their PDLLA/PLCL polymer blends after melt-quenching and treated them at $37^{\circ} \mathrm{C}$ for different aging times. E: elastic modulus calculated as Young's modulus, $\sigma_{\text {max }}$ : Tensile strength, $\varepsilon_{u}$ : Elongation at break.

*Secant modulus at $50 \%$ of deformation.

\begin{tabular}{|c|c|c|c|c|}
\hline & $\begin{array}{c}\mathrm{t}_{\mathrm{a}} \\
\text { (months) }\end{array}$ & $\begin{array}{c}\mathrm{E} \\
(\mathrm{MPa})\end{array}$ & $\begin{array}{c}\sigma_{\max } \\
(\mathrm{MPa})\end{array}$ & $\begin{array}{c}\varepsilon_{\mathrm{u}} \\
(\%)\end{array}$ \\
\hline \multirow{3}{*}{ PDLLA } & 0 & $1023 \pm 100$ & $34 \pm 9$ & $4 \pm 2$ \\
\hline & 1 & $1072 \pm 10$ & $37 \pm 2$ & $5 \pm 1$ \\
\hline & 2 & $1116 \pm 48$ & $32 \pm 2$ & $3 \pm 1$ \\
\hline \multirow{3}{*}{8020} & 0 & $942 \pm 48$ & $38 \pm 3$ & $314 \pm 40$ \\
\hline & 1 & $914 \pm 42$ & $42 \pm 2$ & $9 \pm 1$ \\
\hline & 2 & $958 \pm 16$ & $38 \pm 1$ & $5 \pm 1$ \\
\hline \multirow{3}{*}{6040} & 0 & $606 \pm 27$ & $36 \pm 3$ & $452 \pm 22$ \\
\hline & 1 & $640 \pm 6$ & $30 \pm 2$ & $265 \pm 3$ \\
\hline & 2 & $678 \pm 28$ & $24 \pm 1$ & $22 \pm 1$ \\
\hline \multirow{3}{*}{5050} & 0 & $256 \pm 15$ & $37 \pm 2$ & $554 \pm 53$ \\
\hline & 1 & $484 \pm 7$ & $24 \pm 1$ & $273 \pm 13$ \\
\hline & 2 & $488 \pm 21$ & $21 \pm 1$ & $26 \pm 3$ \\
\hline \multirow{3}{*}{4060} & 0 & $49 \pm 4$ & $35 \pm 5$ & $610 \pm 53$ \\
\hline & 1 & $334 \pm 15$ & $22 \pm 1$ & $317 \pm 32$ \\
\hline & 2 & $355 \pm 2$ & $18 \pm 1$ & $63 \pm 1$ \\
\hline \multirow{3}{*}{2080} & 0 & $4 \pm 1$ & $32 \pm 3$ & $733 \pm 38$ \\
\hline & 1 & $145 \pm 12$ & $22 \pm 2$ & $444 \pm 18$ \\
\hline & 2 & $159 \pm 6$ & $16 \pm 1$ & $327 \pm 45$ \\
\hline \multirow{3}{*}{ PLCL } & 0 & $2 \pm 1 *$ & $25.5 \pm 1$ & $837 \pm 52$ \\
\hline & 1 & $66 \pm 4$ & $17 \pm 1$ & $507 \pm 66$ \\
\hline & 2 & $73 \pm 6$ & $14 \pm 2$ & $538 \pm 31$ \\
\hline
\end{tabular}

As can be observed in Table 1, the elastic modulus increases while the maximum stress and elongation at break decrease with the aging time. The increase of stiffness is much noticeable for composition having more than $50 \mathrm{wt} \%$ of PLCL. This result indicates that the behavior of these blends changed from elastomeric (see Fig. 2.a) to thermoplastic one. Moreover, the elongation at break values suffer a detrimental decrease when blends are subjected to $37^{\circ} \mathrm{C}$ for 2 months. For example, for 8020 composition the initial value decreases from 314\% to 5\%; which is close to the original value of PDLLA. The 6040 and 5050 compositions lost the ductility gained initially showing $\varepsilon_{u}$ values of $22 \%$ and $26 \%$, respectively. After two months at $37{ }^{\circ} \mathrm{C}$ only the 2080 composition showed a close value of $\varepsilon_{\mathrm{u}}$ of that obtained initially by adding $20 \mathrm{wt}$. $\%$ of PLCL to PDLLA. Mechanical properties analysis indicate that PDLLA/PLCL blends change their behavior from being tough to being more brittle.

In order to get better interpretation of the blend morphology, surfaces after tensile fractures of PDLLA/PLCL blends were observed by means of Scanning Electron Microscopy (SEM). The most representative micrographs obtained for 8020 , 5050, and 2080 composition are shown in Table 2. 
Table 2: SEM micrographs of the tensile fractured surfaces of unaged and 2 month aged PDLLA/PLCL 8020, 5050 and 2080 blends.

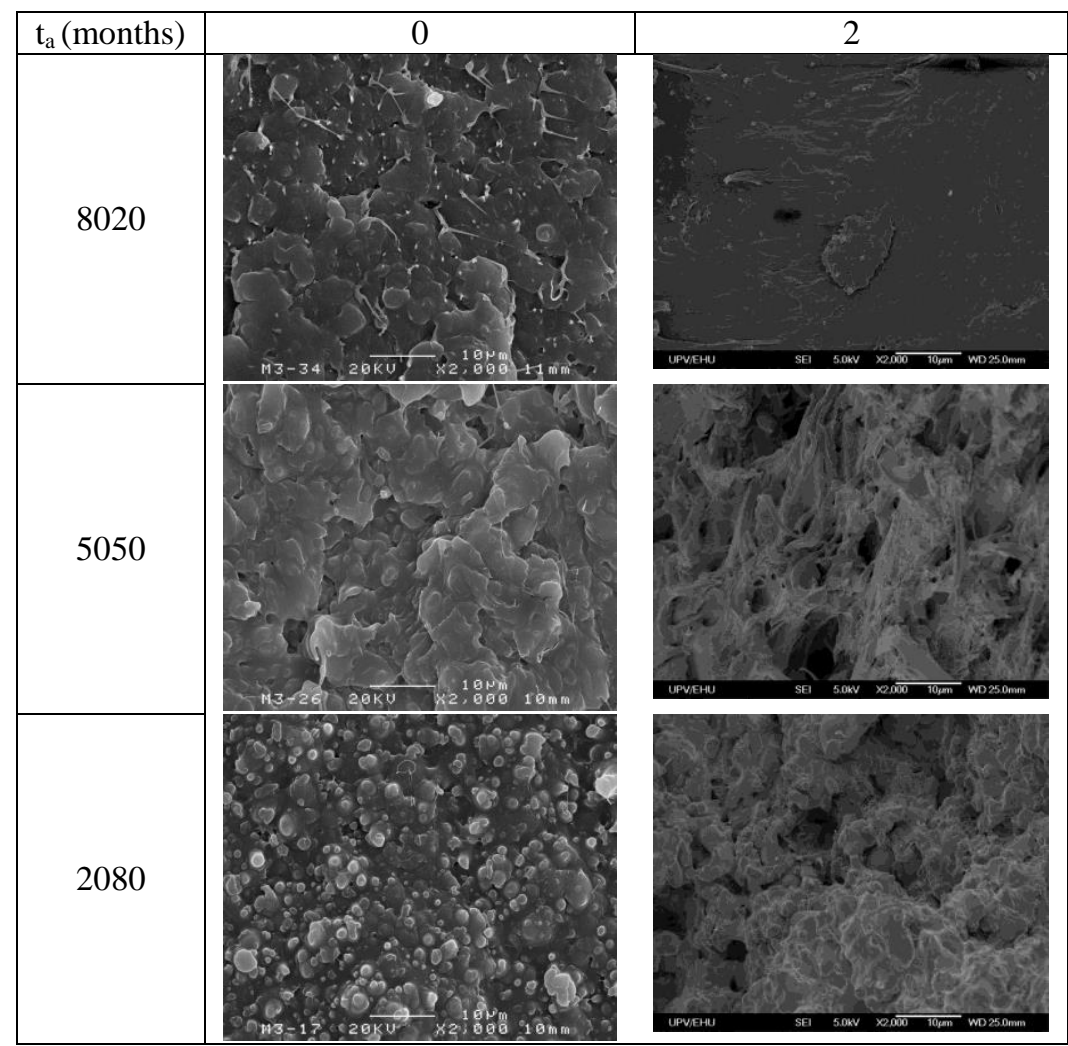

For unaged PDLLA/PLCL blends two phases can be clearly discerned, discrete domains in a continuous matrix, which is in accordance with the immiscibility observed by DSC. It has to be pointed out that no clear distinct interfaces are observed. This could be an indicative of a good interfacial compatibility between the two phases. This good compatibility may be the reason of the improved ductility shown initially by these phase separated blends. The change in mechanical properties is also visible in these micrographs. While unaged PDLLA/PLCL samples show a rough surface as a result of the plastic deformation underwent during the tensile tests, aged samples show a smoother fracture surface typical of brittle polymers. This change is evident if compare the unaged and aged micrographs of 8020 and 5050 compositions as they convert to more brittle samples as mentioned before. However, the 2080 composition preserves the rough surface indicating its capability to deform plastically which is in agreement with the values obtained in Table 1.

\section{Conclusion}

In this work the phase behavior and the evolution of mechanical properties at body temperature of PDLLA/PLCL blends were presented and discussed.

The miscibility state was evaluated according to the unique $T_{\mathrm{g}}$ criterion by means of DSC resulting in a immiscible polymeric blend. The toughness of PDLLA was improved by the addition of PLCL copolymer due to the good interfacial compatibility found by SEM between the two phases. However, PDLLA/PLCL blends suffer a significant change in their mechanical behaviour from elastomeric or ductile to brittle when subjected to $37^{\circ} \mathrm{C}$ for only two months.

Therefore, although more precise measurements are required for full understanding of the mechanical response of this PDLLA/PLCL blend system at service, these preliminary results shed light on the impossibility of some of these blend compositions to be used as orthopaedic implants for bone fracture fixations. 


\section{Acknowledgements}

The authors are grateful for funds from the Basque Government (GV/EJ) Department of Education, Universities and Research for project GIC13/161-IT-632-13 and for a pre-doctoral grant of J. M. U, MICINN for project BIO2010-21542C02-01, and the University of the Basque Country for project UFI11/56.

\section{References}

[1] J. Wolff, The Law of Bone Remodelling. Berlin Heidelberg: Springer-Verlag, 1986.

[2] N. Ashammakhi et al, "Spotlight on naturally absorbable osteofixation devices," J Craniofac Surg, vol. 14, no. 2, pp. 247-259, 2003.

[3] G. O. Hofmann, "Biodegradable implants in traumatology: a review on the state-of-the-art," Arch Orthop Trauma Surg, vol. 114, no. 3, pp. 123-132, 1995.

[4] R. K. Kulkarni et al, "Polylactic acid for surgical implants," Arch Surg, vol. 93, no. 5, pp. 839-843, 1966.

[5] R. R. Bos et al, "Bioabsorbable poly(L-lactide) osteosynthesis plates and screws for the fixation of zygomatic bone fractures," Dtsch Z Mund Kiefer Gesichtschir, vol. 13, no. 6, pp. 422-424, 1989.

[6] R. R. Bos et al, "Bone-plates and screws of bioabsorbable poly (L-lactide)--an animal pilot study," $\mathrm{Br} J \mathrm{Oral}$ Maxillofac Surg, vol. 27, no. 6, pp. 467-476, 1989.

[7] K. L. Gerlach, "In vivo and clinical evaluations of poly(L-lactide) plates and screws for use in maxillofacial traumatology," Clin. Mater., vol. 13, no. 1-4, pp. 21-28, 1993.

[8] K. Bessho, T. Iizuka and K. Murakami, "A bioabsorbable poly-L-lactide miniplate and screw system for osteosynthesis in oral and maxillofacial surgery," J Oral Maxillofac Surg, vol. 55, no. 9, pp. 941-5; 945-6, 1997.

[9] T. Suzuki et al, "Resorbable poly-L-lactide plates and screws for the treatment of mandibular condylar process fractures: a clinical and radiologic follow-up study," J Oral Maxillofac Surg, vol. 62, no. 8, pp. 919-924, 2004.

[10] M. B. Habal and W. S. Pietrzak, "Key points in the fixation of the craniofacial skeleton with absorbable biomaterial," J Craniofac Surg, vol. 10, no. 6, pp. 491-499, 1999.

[11] A. P. Gupta and V. Kumar, "New emerging trends in synthetic biodegradable polymers - Polylactide: A critique," Eur. Polym. J., vol. 43, no. 10, pp. 4053-4074, 2007. DOI: 10.1016/j.eurpolymj.2007.06.045.

[12] A. G. Andreopoulos, E. Hatzi and M. Doxastakis, "Synthesis and properties of poly(lactic acid)," J. Mater. Sci. Mater. Med., vol. 10, no. 1, pp. 29-33, 1999.

[13] M. Hiljanen-Vainio et al, "Modification of poly(L-lactides) by blending: mechanical and hydrolytic behavior," Macromol. Chem. Phys., vol. 197, no. 4, pp. 1503-1523, 1996.

[14] M. H. Baums et al, "Intraarticular migration of a broken biodegradable interference screw after anterior cruciate ligament reconstruction," Knee Surg Sports Traumatol Arthrosc, vol. 14, no. 9, pp. 865-868, 2006.

[15] F. A. Krappel, E. Bauer and U. Harland, "The migration of a BioScrew as a differential diagnosis of knee pain, locking after ACL reconstruction: a report of two cases," Arch Orthop Trauma Surg, vol. 126, no. 9, pp. 615-620, 2006.

[16] B. Lembeck and N. Wulker, "Severe cartilage damage by broken poly-L-lactic acid (PLLA) interference screw after ACL reconstruction," Knee Surg Sports Traumatol Arthrosc, vol. 13, no. 4, pp. 283-286, 2005.

[17] M. Malin et al, "Biodegradable lactone copolymers. II. Hydrolytic study of $\varepsilon$-caprolactone and lactide copolymers," $J$ Appl Polym Sci, vol. 59, no. 8, pp. 1289-1298, 1996.

[18] D. W. Grijpma, G. J. Zondervan, A. J. Pennings, "High molecular weight copolymers of L-lactide and Î $\mu$-caprolactone as biodegradable elastomeric implant materials," Polym. Bull. (Berlin), vol. 25, no. 3, pp. 327-333, 1991.

[19] G. Perego, T. Vercellio and G. Balbontin, "Copolymers of L- and D,L-lactide with 6-caprolactone: synthesis and characterization," Die Makromolekulare Chemie, vol. 194, no. 9, pp. 2463-2469, 1993.

[20] L. A. Utracki, "Thermodynamics of polymer blends," in L. A. Ultracki, ed. 2002.

[21] M. E. Broz, D. L. VanderHart and N. R. Washburn, "Structure and mechanical properties of poly(D,L-lactic acid)/poly(E-caprolactone) blends," Biomaterials, vol. 24, no. 23, pp. 4181-4190, 2003.

[22] H. Tsuji and Y. Ikada, "Blends of aliphatic polyesters. I. Physical properties and morphologies of solution-cast blends from poly(DL-lactide) and poly(epsilon-caprolactone)," J. Appl. Polym. Sci., vol. 60, no. 13, pp. 2367-2375, 1996.

[23] R. Dell'Erba et al, "Immiscible polymer blends of semicrystalline biocompatible components: thermal properties and phase morphology analysis of PLLA/PCL blends," Polymer, vol. 42, no. 18, pp. 7831-7840, 2001. 
[24] V. Vilay et al, "Characterization of the mechanical and thermal properties and morphological behavior of biodegradable poly(L-lactide)/poly(.vepsiln.-caprolactone) and poly(L-lactide)/poly(butylene succinate-co-L-lactate) polymeric blends," J. Appl. Polym. Sci., vol. 114, no. 3, pp. 1784-1792, 2009.

[25] N. Lopez-Rodriguez et al, "Crystallization, morphology, and mechanical behavior of polylactide/poly(E-caprolactone) blends," Polym. Eng. Sci., vol. 46, no. 9, pp. 1299-1308, 2006. 